\title{
Functional Outcomes among a Cohort of Children in Northeastern Brazil Meeting Criteria for Follow-Up of Congenital Zika Virus Infection
}

\author{
Jeanne Bertolli, ${ }^{1 \star}$ Jacob Elijah Attell, ${ }^{2}$ Charles Rose,${ }^{3}$ Cynthia A. Moore,${ }^{3}$ Flávio Melo, ${ }^{4}$ Jennifer Erin Staples, ${ }^{5}$ Kim Kotzky, ${ }^{6}$ \\ Nevin Krishna, ${ }^{7}$ Ashley Satterfield-Nash, ${ }^{6}$ Isabela Ornelas Pereira, ${ }^{8}$ André Pessoa, ${ }^{9}$ Donna Camille Smith, ${ }^{10}$ \\ Ana Carolina Faria e Silva Santelli, ${ }^{11}$ Coleen A. Boyle, ${ }^{3}$ and Georgina Peacock ${ }^{1}$ \\ ${ }^{1}$ Division of Human Development and Disability, Centers for Disease Control and Prevention (CDC), Atlanta, Georgia; ${ }^{2}$ Eagle Global Scientific, San \\ Antonio, Texas; ${ }^{3}$ National Center on Birth Defects and Developmental Disabilities, CDC, Atlanta, Georgia; ${ }^{4}$ Hospital Regional de Guarabiral \\ Governo do Estado da Paraíba, João Pessoa, Brazil; ${ }^{5}$ Division of Vector-Borne Diseases, CDC, Fort Collins, Colorado; ${ }^{6}$ Oak Ridge Institute for \\ Science and Education, Oak Ridge, Tennessee; ${ }^{7}$ Office of Public Health Preparedness and Response, CDC, Atlanta, Georgia; ${ }^{8}$ Transmissible \\ Diseases Department (DEVIT), Ministry of Health of Brazil, Brasilia, Brazil; ${ }^{9}$ Hospital Infantil Albert Sabin, Fortaleza, Brazil; ${ }^{10}$ Division of Congenital \\ and Developmental Disorders, CDC, Atlanta, Georgia; ${ }^{11}$ Division of Global HIV and TB, Center for Global Health, CDC, Atlanta, Georgia
}

Abstract. Following the large outbreak of Zika virus in the Western Hemisphere, many infants have been born with congenital Zika virus infection. It is important to describe the functional outcomes seen with congenital infections to allow for their recognition and appropriate interventions. We evaluated 120 children conceived during the 2015-2016 Zika virus outbreak in Paraíba, Brazil, who were approximately 24 months old, to assess functional outcomes. All children met either anthropometric criteria or laboratory criteria suggestive of possible congenital Zika virus infection. We collected results of previous medical evaluations, interviewed parents, and performed physical examinations and functional assessments, for example, the Hammersmith Infant Neurological Examination (HINE). We compared patterns of neurologic outcomes and developmental delay at age 24 months by whether children met anthropometric or laboratory criteria, or both. Among children meeting both criteria, 60\% (26/43) were multiply affected (had severe motor impairment, severe developmental delay, and suboptimal HINE scores), compared with $5 \%(3 / 57)$ meeting only laboratory criteria and none (0/20) meeting only anthropometric criteria. Of the remaining 91 children, 49\% (45) had developmental delay, with more severe delay seen in children meeting both criteria. Although children meeting physical and laboratory criteria for potential congenital Zika virus infection were more severely affected, we did identify several children with notable adverse neurologic outcomes and developmental delay with no physical findings but potential laboratory evidence of Zika virus infection. Given this, all children who were potentially exposed in utero to Zika virus should be monitored in early childhood for deficits to allow for early intervention.

\section{INTRODUCTION}

Zika virus is believed to have been introduced in Brazil in 2013. ${ }^{1-3}$ However, it was not until August 2015, when healthcare providers in the state of Pernambuco noticed an unusual increase in newborns with microcephaly, that Zika virus was identified to cause congenital infections with serious outcomes. ${ }^{4}$ Although birth defects including microcephaly were the earliest recognized features of congenital Zika virus infection, ${ }^{5-11}$ later findings revealed that the extent of damage caused by intrauterine Zika virus infection is not always apparent at birth ${ }^{10-12}$ but can manifest later, ${ }^{11,13-15}$ raising the possibility that cases could be missed.

The increased incidence of severe microcephaly that first brought Zika virus to attention in Brazil has been likened to "the tip of the iceberg,"16,17 yet the size and hallmarks of the rest of the presumed iceberg have been difficult to ascertain in part because of diagnostic challenges. ${ }^{18}$ Aragao et al. ${ }^{17}$ used neuroimaging to describe a spectrum of congenital infections that includes three levels of severity: microcephaly at birth, postnatal microcephaly, and without microcephaly. Other research suggests the possibility of impaired health and development in children without obvious manifestations of congenital infection at birth. ${ }^{11,12}$ Subtle and delayed findings have been identified with congenital Zika virus infection, but their relation to functional deficits is unclear. Few studies have investigated functional outcomes of congenital Zika virus exposure among children aged 12 months or older, born with or without birth

\footnotetext{
*Address correspondence to Jeanne Bertolli, Division of Human Development and Disability, Centers for Disease Control and Prevention (CDC), 1600 Clifton Rd., Mailstop E88, Atlanta, GA 30329. E-mail: jub7@cdc.gov
}

defects, ${ }^{14,19}$ and none presented outcomes in relation to infant Zika virus laboratory test results. We followed up a group of infants who were conceived during the 2015-2016 Zika virus outbreak in northeastern Brazil and had been evaluated at 17 months of age for evidence of congenital Zika infection ${ }^{20}$ to characterize their functional outcomes at approximately 24 months of age, including neuromotor function, vision, hearing, and developmental delay.

\section{MATERIALS AND METHODS}

Population. The Brazilian Ministry of Health, the State Health Secretariat of Paraíba, and the U.S. CDC collaborated on the Zika Outcomes and Development in Infants and Children (ZODIAC) investigation. Infants who were conceived during the 2015-2016 Zika virus outbreak in northeastern Brazil and had participated in a 2016 case-control study to investigate the association between microcephaly and congenital Zika infection ${ }^{20}$ were eligible to participate if they met either laboratory or anthropometric criteria.

1. Laboratory criteria: A nonnegative test for Zika-specific neutralizing antibodies in an infant sample obtained at 1-7 months of age.

2. Anthropometric criteria: Head circumference $(\mathrm{HC}) \leq 3 \mathrm{rd}$ percentile for gestational age and sex or $\mathrm{HC}>3$ rd percentile for gestational age and sex and $\mathrm{HC}$ :body length $\leq 0.65$ (i.e., disproportionately small $\mathrm{HC}$, given the length). Note: disproportionate measurements were included as inclusion criteria in the initial case-control study based on early observations of severely impacted infants and later supported by observations of affected fetuses. ${ }^{21}$ 
We further restricted eligibility for the ZODIAC follow-up investigation to children living in certain areas (i.e., macroregions 1 and 2) of Paraíba state because of logistical constraints. This report includes 19 children with microcephaly and laboratory criteria previously reported by Satterfield-Nash et al. ${ }^{22}$

Objective. Our primary objective was to describe the varying severity of functional outcomes that might be associated with congenital Zika virus infection. The protocol for this investigation was approved by the Brazilian National Ethics Committee. The parents who were respondents for this investigation provided informed consent for their own and their child's participation.

Assessments. We analyzed data from three time points, namely, birth, 1-7 months of age, and 19-26 months of age (Table 1). For the investigation at 19-26 months of age, information was collected from medical records, study assessments of the children, and interviews with their caregivers. Information from medical records included $\mathrm{HC}$, length, and weight, reported as $z$-scores and SDs on international growth parameter distributions. ${ }^{23,24}$ The dysmorphologist (CAM) who was on the research team for both the original 2016 case-control study and the ZODIAC study rereviewed photographs of the children to confirm typical Zika phenotypes $^{8}$ or nonspecific dysmorphic features. Licensed physicians performed growth, ophthalmologic, and physical examinations, and a neurologic assessment, and referred children for further evaluation as indicated. Children's blood was drawn and tested for other factors associated with developmental delay, including blood lead, hematocrit, and thyroid hormone (T4) level.

Physicians were trained to implement the Hammersmith Infant Neurological Examination (HINE), a standardized neurologic examination that has been validated for ages 324 months, to assess neuromotor function and visual and auditory responses. ${ }^{25}$ Trained interviewers administered instruments used to collect caregiver-reported data, including a seizure screener ${ }^{26}$ and the Ages and Stages Questionnaires (ASQ-3), ${ }^{27}$ a series of 21 questionnaires designed to screen the developmental performance of children in five domains (http://agesandstages.com). For severely affected children, we used an amended protocol to administer ASQ-3 questionnaires that were appropriate to a child's development instead of beginning the assessment with a questionnaire designed for a child of the same biological age (see Supplemental Appendix for details). Field investigators entered data using Research Electronic Data Capture, a secure Web application. ${ }^{28}$

Analyses. We compared developmental and neurologic outcomes of children based on whether they met the anthropometric or laboratory criteria of possible congenital infection or both. Children were considered to meet the anthropometric criteria if their $\mathrm{HC}$ or $\mathrm{HC}$ :length ratio fell within the range described earlier. Children had been tested at the age of 17 months for evidence of Zika virus infection as part of the previously conducted case-control study. ${ }^{20}$ Given the timing of sample collection, serologic testing (i.e., Zika virus IgM ELISA and Zika and dengue virus plaque reduction neutralization testing) was performed; no nucleic acid testing was performed on the postnatal samples. Children were considered to meet the laboratory criteria if they had a nonnegative test for Zika neutralizing antibodies (confirmed, presumed, or possible result). For the purpose of this study, Zika virus infection was considered confirmed if an infant blood specimen tested positive for Zika virus-associated IgM antibodies with evidence of neutralizing antibodies against Zika virus. Infection was presumed if an infant sample tested negative for Zika virus-specific IgM antibodies, with Zika virus-dengue virus ratio of maternal-infant neutralizing antibody titers $<1 .{ }^{20}$ Infection was possible if an infant sample tested negative for Zika virus-specific lgM antibodies, with neutralizing antibodies against Zika virus that did not meet the presumed definition.

TABLE 1

Variables, sources, and time frames; Zika Outcomes and Development in Infants and Children investigation, Paraíba, Brazil, 2017

\begin{tabular}{|c|c|c|}
\hline Variable & Source & Child age when assessed \\
\hline \multicolumn{3}{|l|}{ Demographics } \\
\hline Child & ZODIAC assessment (medical record abstraction) & 19-26 months \\
\hline Child & Case-control records (medical record and assessment) & Birth and 1-7 months \\
\hline Caregiver & ZODIAC assessment (self-report) & $19-26$ months \\
\hline \multicolumn{3}{|l|}{ Laboratory evidence of infection } \\
\hline Child & $\begin{array}{l}\text { Case-control (Zika and dengue lgM antibody and } \\
\text { neutralization antibody testing of infant specimens) }\end{array}$ & $1-7$ months \\
\hline \multicolumn{3}{|l|}{ Medical outcomes } \\
\hline Dysmorphic features & Case-control (expert clinical evaluation) & 1-7 months \\
\hline Anthropometric parameters & ZODIAC (medical record and assessment) and Case-control records & $\begin{array}{l}\text { Birth, } 1-7 \text { months, and } \\
\text { 19-26 months }\end{array}$ \\
\hline Neuromotor function & $\begin{array}{l}\text { ZODIAC (physician assessment, Hammersmith Infant Neurological } \\
\text { Examination }{ }^{25} \text { ) }\end{array}$ & $19-26$ months \\
\hline Cerebral palsy & ZODIAC (physician assessment) & 19-26 months \\
\hline Hearing impairment & $\begin{array}{l}\text { ZODIAC (physician assessment and abstraction of diagnostic evaluation } \\
\text { results) }\end{array}$ & $\begin{array}{l}\text { Approximately } 19-26 \\
\text { months }\end{array}$ \\
\hline $\begin{array}{l}\text { Vision impairment (abnormalities of } \\
\text { retina and fixation/following) }\end{array}$ & $\begin{array}{l}\text { ZODIAC (physician assessment and functional vision and ophthalmologic } \\
\text { examination) }\end{array}$ & 19-26 months \\
\hline Seizures & ZODIAC assessment (parent report and seizure screener) & 19-26 months \\
\hline $\begin{array}{l}\text { Other medical conditions and } \\
\text { hospitalization }\end{array}$ & ZODIAC (medical record abstraction) & $\leq 19$ months \\
\hline \multicolumn{3}{|l|}{ Developmental delay } \\
\hline $\begin{array}{l}\text { Developmental quotient and } \\
\text { developmental age equivalent }\end{array}$ & $\begin{array}{l}\text { ZODIAC assessment (parent report and Ages and Stages Questionnaires, } \\
\text { third edition }{ }^{27} \text { ) }\end{array}$ & 19-26 months \\
\hline
\end{tabular}


Developmental delay was measured by the developmental quotient $z$-score (DQz) for each of the five ASQ-3 domains: communication, gross motor skills, fine motor skills, problem solving, and personal-social skills. The developmental quotient (DQ) was defined as the ratio of the child's developmental (functional) age, determined based on ASQ-3 scores, and the child's biological age, multiplied by 100 . We calculated an ASQ $z$-score for each child on each domain by comparing the child's score on the questionnaire for that domain to the mean and SD of the distribution of the scores of a large number of Brazilian children, drawn from child daycare centers serving low-income families, who received the same questionnaire. ${ }^{29}$ We used these ASQ z-scores to obtain a DQ and a DQz for each child on each domain. Method details are provided in the Supplemental Appendix.

Three developmental delay groups were identified for each of the five ASQ-3 domains, according to children's ASQ-3 scores and DQz cutoffs: 1) no delay (z-score above -1 SD from the mean), 2) possible delay (z-score from -1 to $-2 \mathrm{SD}$ ), and 3) likely delay (z-score below -2 SD), according to convention. ${ }^{27}$ For this study, the degree of overall developmental delay incorporating scores on all five ASQ domains was described as follows: 1) none: above $-1 \mathrm{SD}$ on all five $A S Q-3$ domains, 2) severe: below -2 SD on at least two domains, and 3) mild to moderate: the remaining combinations, that is, $z$-scores below -2 SD on one domain or from -1 SD to -2 SD on at least one domain.

Recognizing that some congenitally infected children may have subtle findings that are not distinctive for congenital Zika infection, ${ }^{14,15,19}$ we looked for patterns of functional outcomes by the anthropometric or laboratory criteria, or both. We examined proportions of children with developmental delay (mild to moderate or severe) on any ASQ-3 domain, with and without a neurologic deficit (i.e., severe motor impairment, cerebral palsy of any type, a suboptimal HINE score on any domain, functional vision problems, a positive seizure screen) within and across subgroups (anthropometric, laboratory, or both). To investigate whether the findings varied by strength of laboratory evidence that supported congenital Zika virus infection versus passive maternal transfer of neutralizing antibodies, we further subdivided children and analyzed the outcome data according to their original laboratory classification. ${ }^{20}$ We hypothesized that children with congenital Zika infection might have a pattern of domain-specific developmental delay, so we also examined children with developmental delay in each of the five ASQ-3 domains by the anthropometric and laboratory criteria.

\section{RESULTS}

Of the 592 children included in the 2016 case-control study, 273 were eligible for follow-up (Figure 1). Overall, 122 children (45\%) participated in the follow-up investigation, 76 (28\%) were lost to follow-up, and 75 families (28\%) refused to participate. Two children were excluded from analysis because they were missing critical data, leaving 120 children who were included based on the anthropometric and/or laboratory criteria and assessed from July to October 2017. At the time of evaluation for the ZODIAC investigation, the median age was 23 months (range, 19-26 months). Table 2 presents demographic information on the children and their caregivers.

Forty-three (36\%) children met both anthropometric and laboratory criteria, including 27 children who had

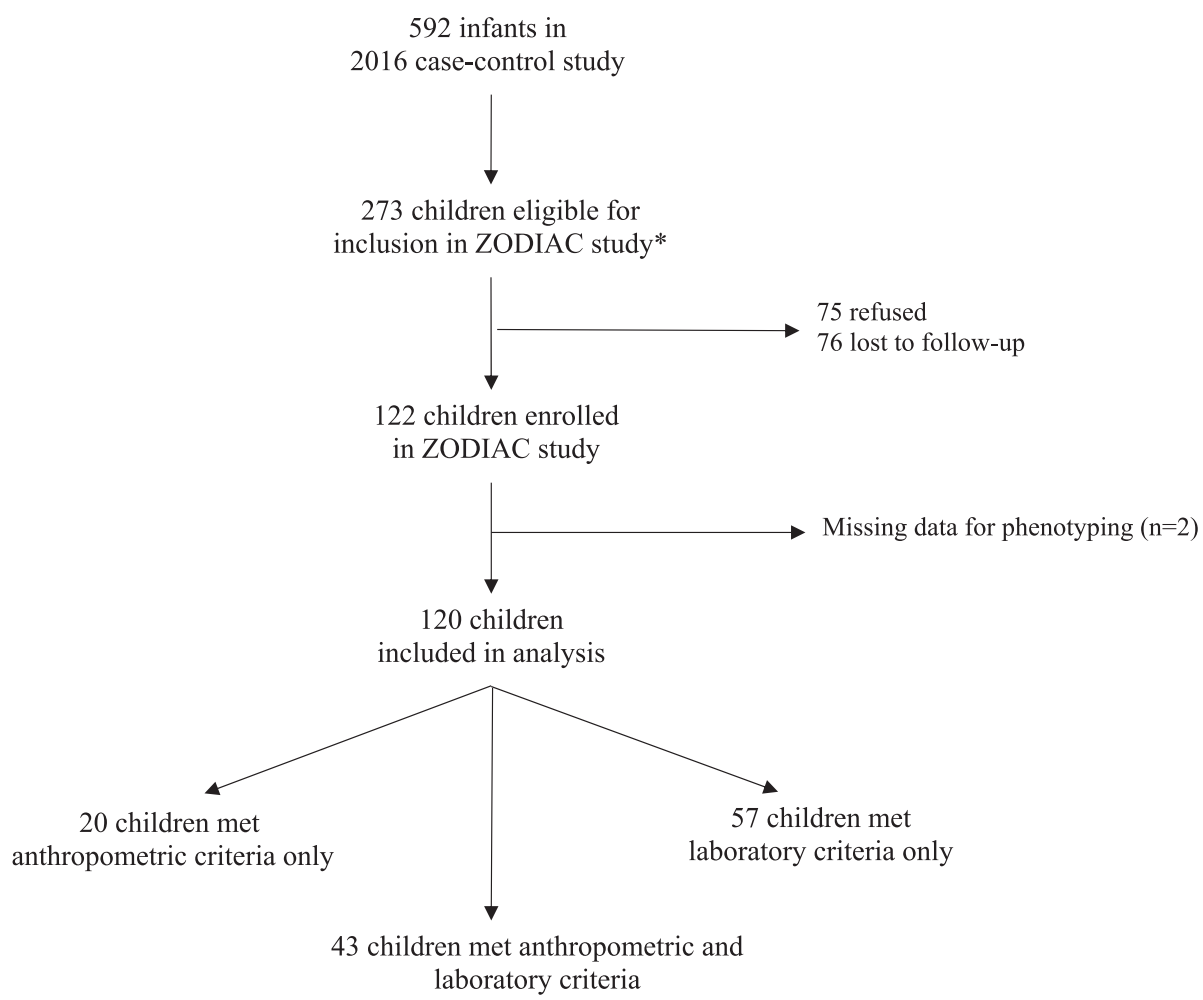

FIGURE 1. Eligibility for the Zika Outcomes and Development in Infants and Children investigation, Paraíba, Brazil, 2017. Anthropometric criteria: head circumference $(\mathrm{HC}) \leq 3$ rd percentile for gestational age and sex or $\mathrm{HC}>3$ rd percentile for gestational age and sex and $\mathrm{HC}$ : body length $\leq 0.65$; Laboratory criteria: non-negative Zika neutralizing antibody test results in an infant sample. 
TABLE 2

Demographic characteristics of 120 children meeting anthropometric and/or laboratory criteria for follow-up of congenital Zika virus infection, and of their caregivers and families, Paraíba, Brazil, 2017

\begin{tabular}{|c|c|c|}
\hline Characteristic & Number & Percent \\
\hline \multicolumn{3}{|l|}{ Child's sex } \\
\hline Male & 60 & 50 \\
\hline Female & 60 & 50 \\
\hline \multicolumn{3}{|c|}{ Child's age at assessment (months) } \\
\hline $19-20$ & 11 & 9 \\
\hline $21-22$ & 49 & 41 \\
\hline $23-24$ & 46 & 38 \\
\hline $25-26$ & 14 & 12 \\
\hline \multicolumn{3}{|c|}{ Caregiver relationship with the child } \\
\hline Mother & 115 & 96 \\
\hline Other caregiver* & 5 & 4 \\
\hline \multicolumn{3}{|l|}{ Caregiver race } \\
\hline White & 14 & 12 \\
\hline Black & 15 & 13 \\
\hline Brown & 81 & 68 \\
\hline Yellow & 8 & 7 \\
\hline Indigenous & 2 & 2 \\
\hline \multicolumn{3}{|l|}{ Caregiver age (years) } \\
\hline$\leq 18$ & 8 & 7 \\
\hline $19-23$ & 29 & 24 \\
\hline $24-28$ & 23 & 19 \\
\hline $29-33$ & 34 & 28 \\
\hline$>33$ & 26 & 22 \\
\hline \multicolumn{3}{|c|}{ Caregiver education (years) } \\
\hline$\leq 6$ & 24 & 20 \\
\hline $7-8$ & 24 & 20 \\
\hline $9-11$ & 36 & 30 \\
\hline$\geq 12$ & 36 & 30 \\
\hline \multicolumn{3}{|c|}{ Monthly family income $(\mathrm{R} \$) \dagger$} \\
\hline$<500$ & 27 & 23 \\
\hline $500-1,499$ & 69 & 58 \\
\hline$\leq 1,500$ & 18 & 15 \\
\hline Unknown & 6 & 5 \\
\hline \multicolumn{3}{|c|}{ Trouble covering basic expenses } \\
\hline Never & 38 & 32 \\
\hline Rarely & 31 & 26 \\
\hline Somewhat often & 25 & 21 \\
\hline Very often & 25 & 21 \\
\hline Unknown & 1 & 1 \\
\hline \multicolumn{3}{|l|}{ Household size } \\
\hline $2-4$ & 78 & 65 \\
\hline $5-7$ & 37 & 31 \\
\hline$>8$ & 5 & 4 \\
\hline
\end{tabular}

$\dagger R \$ 500.00=$ US $\$ 154.32$ in 2017.

microcephaly and 16 who were classified as disproportionate. Of the remaining children, $57(48 \%)$ met only the laboratory criteria and $20(17 \%)$ met only the anthropometric criteria for inclusion in the follow-up study; all children with only the anthropometric criteria were disproportionate, and none had microcephaly (Table 3).

Severe motor impairment, cerebral palsy, HINE scores indicating impaired response to auditory stimuli and impaired response to visual stimuli, a suboptimal score on any HINE domain, and a positive screen for seizures were observed in children meeting both anthropometric and laboratory criteria but not among children meeting only anthropometric or laboratory criteria, with the exception of one child in the latter group, who had suboptimal HINE scores without the other outcomes we investigated (Table 3). Retinal abnormalities were observed in seven children (16\%) meeting both criteria compared with none meeting only the anthropometric or laboratory criteria. Also 16 children $(37 \%)$ meeting both criteria were found to have functional vision impairment compared with none in the other two groups.
Of the children meeting both anthropometric and laboratory criteria, $63 \%$ had severe developmental delay, $16 \%$ had mild to moderate delay, and $23 \%$ had no delay, according to ASQ3 assessments. Ten percent (two of 20 children) meeting only the anthropometric criteria and $5 \%$ (three of 57) meeting only the laboratory criteria had severe developmental delay. Nine $(45 \%)$ of 20 children meeting only the anthropometric criteria and $24(42 \%)$ of 57 meeting only the laboratory criteria had mild to moderate developmental delay.

Table 4 shows that among 43 children who met both anthropometric and laboratory criteria, 26 (60\%) were multiply affected with severe motor impairment, severe developmental delay, and multiple suboptimal HINE scores, with or without vision problems. Of these 26 children, 12 (46\%) had laboratory results indicating confirmed Zika virus infection, six (23\%) had presumed Zika virus infection, and eight (31\%) had possible Zika virus infection. All the children with test results indicating confirmed Zika virus infection had microcephaly. One child with these laboratory findings and disproportionate measurements at birth developed microcephaly postnatally. Among 57 children meeting the laboratory criteria only, three (5\%) had adverse neurologic outcomes in addition to developmental delay. One of these children had the same pattern of multiple, severe neurologic outcomes and severe developmental delay described in the multiply affected group meeting both criteria; this child had laboratory results indicating possible infection. The other two children had mild to moderate developmental delay and a positive seizure screen without other adverse neurologic outcomes, and laboratory results indicating possible and presumed infection. None of the 20 children who only had anthropometric features (all with disproportionate $\mathrm{HC}$ :length) were multiply affected.

Developmental delay without other outcomes was noted for eight children (19\%) who met both anthropometric and laboratory criteria (none with laboratory results indicating confirmed infection), compared with 11 (55\%) meeting only the anthropometric criteria and 26 (46\%) meeting only the laboratory criteria. Of children meeting only the laboratory criteria, $46 \%(26 / 57)$ had ASQ-3 scores, indicating mild to moderate developmental delay, including the only child in the "laboratory criteria only" group whose test results indicated confirmed infection; seven (54\%) of 13 with presumed infection; and $18(42 \%)$ of 43 with possible infection (data not shown). Neither developmental delay nor adverse neurologic outcomes were observed for nine $(45 \%)$ of the children meeting only the anthropometric criteria, compared with 28 (49\%) meeting the laboratory criteria only, and nine (21\%) meeting both; all nine of the latter group had possible laboratory evidence of infection.

Domain-specific developmental delay is presented in Figure 2. Children meeting both anthropometric and laboratory criteria were more likely to have overall developmental delay and delay in any specific domain. Similar percentages of children meeting only the laboratory or anthropometric criteria had developmental delay on specific ASQ-3 domains, with the exception of the gross and fine motor domains.

The majority of children in all three categories had a blood lead level above the reference value of $>5 \mu \mathrm{g} / \mathrm{dL}$ : $60 \%$ (12 of 20 ) children meeting the anthropometric criteria only, including six of 11 with developmental delay; $64 \%$ (35 of 55) children meeting the laboratory criteria only, including 17 of 29 with developmental delay; and $70 \%$ (30 of 43 ) children meeting both, 
TABLE 3

Functional outcomes* at approximately 24 months of age, among children meeting anthropometric and/or laboratory criteria for follow-up of congenital Zika virus infection, Paraíba, Brazil, 2017

\begin{tabular}{|c|c|c|c|}
\hline \multirow[b]{3}{*}{ Outcome } & \multicolumn{3}{|c|}{ Anthropometric/laboratory criteria† of congenital Zika infection } \\
\hline & $\begin{array}{l}\text { Microcephaly/disproportionate } \\
\text { with laboratory criteria§ }\end{array}$ & $\begin{array}{l}\text { Disproportionate without } \\
\text { laboratory criteria }\end{array}$ & $\begin{array}{l}\text { Laboratory } \\
\text { criteria only }\end{array}$ \\
\hline & $N=43, N(\%)$ & $N=20, \ddagger N(\%)$ & $N=57, N(\%)$ \\
\hline Severe motor impairment & $26(61)$ & $0(0)$ & $0(0)$ \\
\hline Cerebral palsy & $25(58)$ & $0(0)$ & $0(0)$ \\
\hline Impaired response to auditory stimuli & $23(54)$ & $0(0)$ & $0(0)$ \\
\hline Impaired response to visual stimuli-HINE & $21(49)$ & $0(0)$ & $0(0)$ \\
\hline Suboptimal score on any HINE domain & $26(61)$ & $0(0)$ & $1(2)$ \\
\hline \multicolumn{4}{|l|}{ Developmental delay classification|| (overall) } \\
\hline Severe & $27(63)$ & $2(10)$ & $3(5)$ \\
\hline Mild to moderate & 7 (16) & $9(45)$ & $26(46)$ \\
\hline None & $9(21)$ & $9(45)$ & $28(49)$ \\
\hline \multicolumn{4}{|l|}{ Vision } \\
\hline Retinal abnormalities & 7 (16) & $0(0)$ & $0(0)$ \\
\hline Abnormal fixation and following & $16(37)$ & $0(0)$ & $0(0)$ \\
\hline Positive seizure screen & $18(43)$ & $0(0)$ & $2(4)$ \\
\hline Missing & 1 & 0 & 1 \\
\hline \multicolumn{4}{|c|}{ 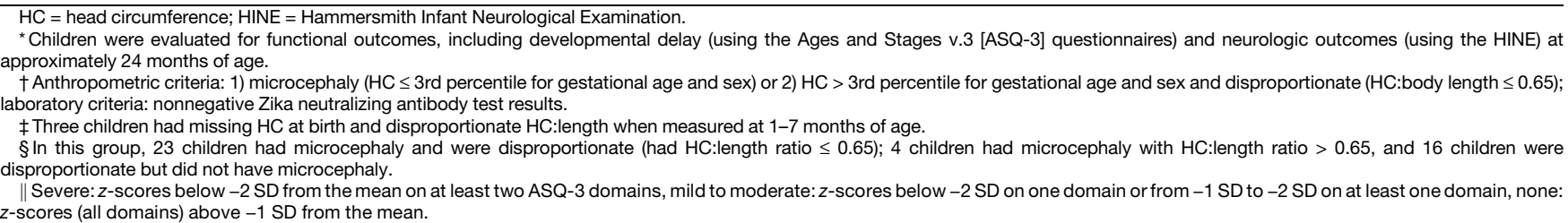 } \\
\hline
\end{tabular}

including 25 of 34 with developmental delay. Hematocrit was below the reference range for six $(30 \%)$ of the children meeting the anthropometric criteria, nine (16\%) meeting the laboratory criteria, and five (12\%) meeting both. None of the children in any of the three groups had a free T4 value below the reference range. Among the 74 children who had developmental delay with or without adverse neurologic outcomes, $16(22 \%)$ had nonspecific dysmorphic features that were consistent neither with the recognized congenital Zika phenotype nor with other recognized congenital syndromes.

\section{DISCUSSION}

Follow-up of children who were conceived during a large Zika virus outbreak and who met anthropometric or laboratory criteria suggesting congenital Zika infection revealed

TABLE 4

Neurologic outcomes and developmental delay ${ }^{\star}$ at approximately 24 months of age, among children meeting the anthropometric and/or laboratory criteria† of congenital Zika virus infection, Paraíba, Brazil, 2017

\begin{tabular}{|c|c|c|c|}
\hline Criteria of congenital Zika virus infection & $\begin{array}{l}\text { Neurologic outcomes } \\
\text { and developmental delay } \neq\end{array}$ & $\begin{array}{l}\text { Developmental } \\
\text { delay only§ }\end{array}$ & $\begin{array}{l}\text { Neither developmental delay } \\
\text { nor neurologic outcomes }\end{array}$ \\
\hline \multicolumn{4}{|c|}{ Microcephaly/disproportionate $\|$ with laboratory criteria $(N=43)$} \\
\hline Total & $26(60)$ & $8(19)$ & $9(21)$ \\
\hline Confirmed & 12 & 0 & 0 \\
\hline Presumed & 6 & 1 & 0 \\
\hline Possible & 8 & 7 & 9 \\
\hline \multicolumn{4}{|l|}{ Laboratory criteria only $(N=57)$} \\
\hline Total & $3(5)$ & $26(46)$ & $28(49)$ \\
\hline Confirmed & 0 & 1 & 0 \\
\hline Presumed & 1 & 7 & 5 \\
\hline Possible & 2 & 18 & 23 \\
\hline \multicolumn{4}{|c|}{ Disproportionate without laboratory criteria $(N=20)$} \\
\hline No evidence of infection & 0 & $11(55)$ & $9(45)$ \\
\hline Total & $29(24)$ & $45(38)$ & $46(38)$ \\
\hline \multicolumn{4}{|c|}{ 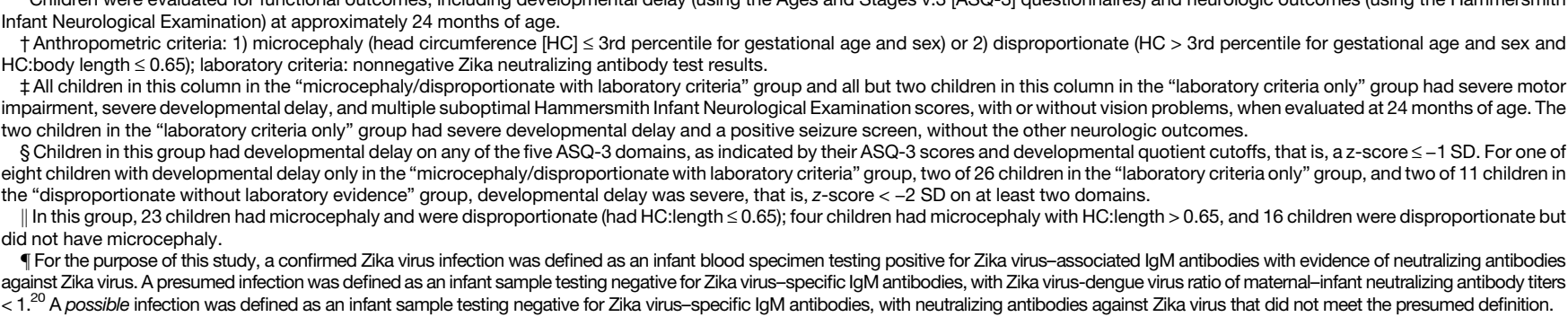 } \\
\hline
\end{tabular}


Overall Developmental Delay

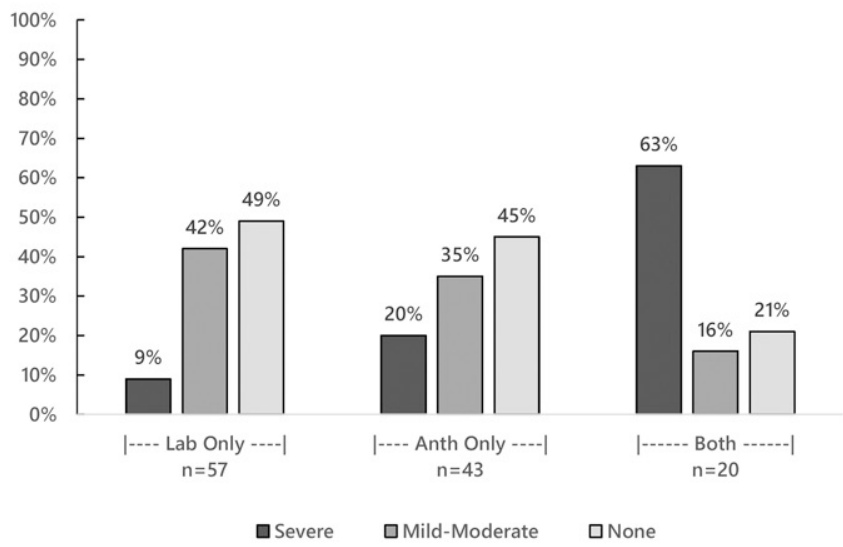

Gross Motor Delay

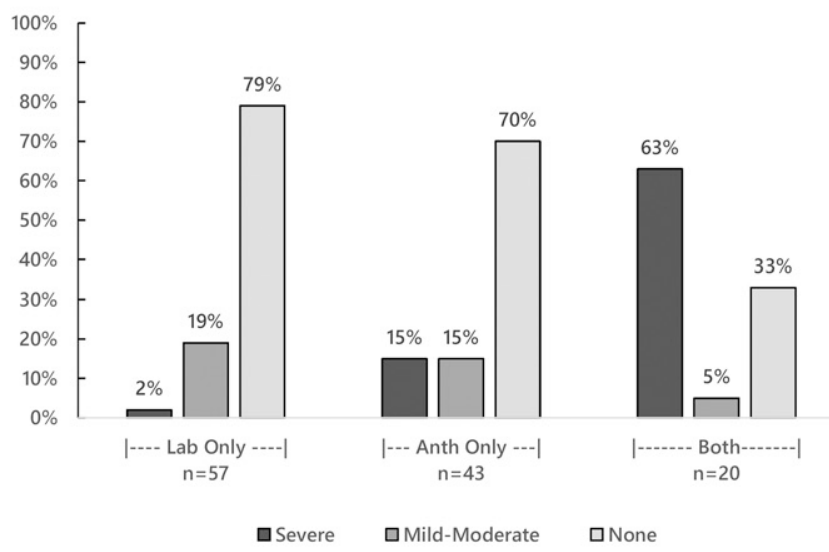

Problem-Solving Delay

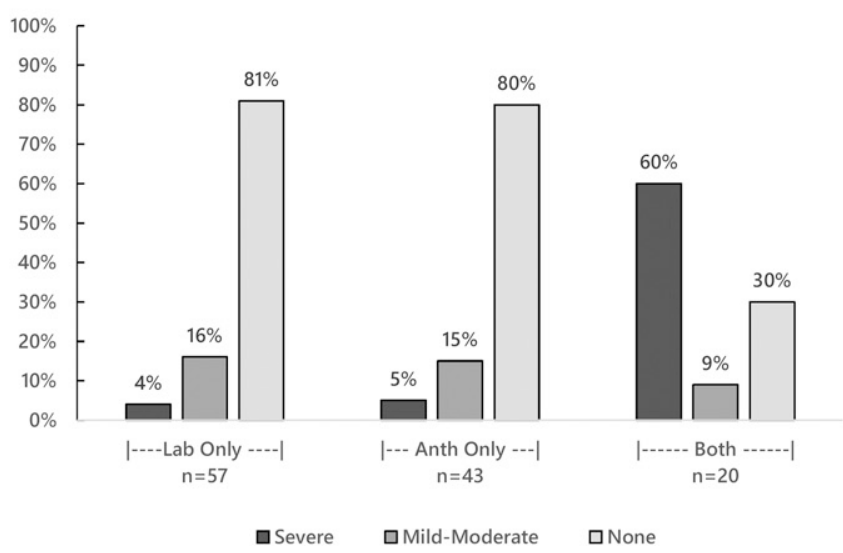

Communication Delay

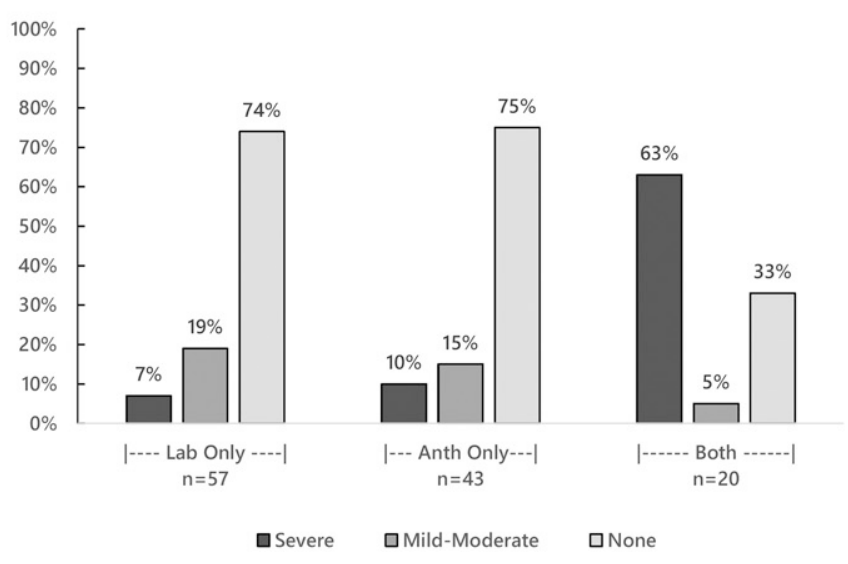

Fine Motor Delay

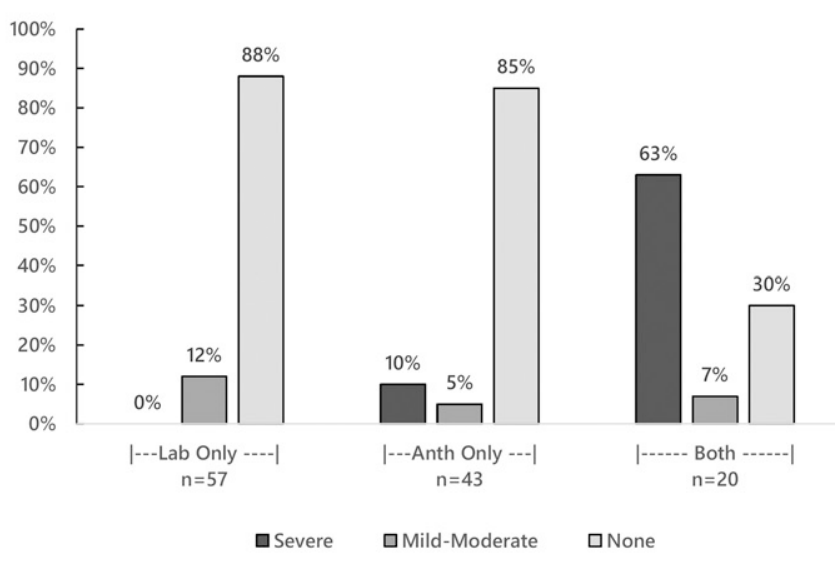

Personal-Social Delay

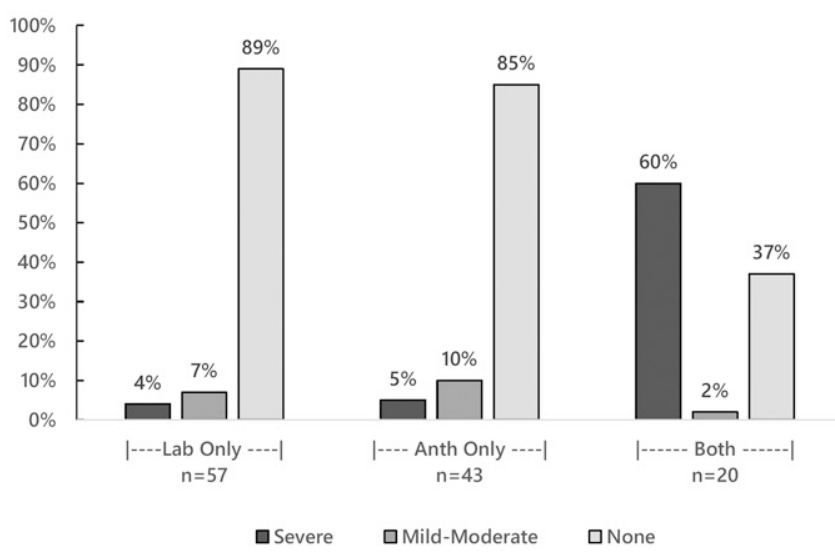

FIGURE 2. Overall developmental delay and domain-specific delay at approximately 24 months of age, among children meeting the anthropometric and/or laboratory criteria for follow-up of congenital Zika virus infection, Paraíba, Brazil, 2017. Anthropometric (Anth) criteria: head circumference $(\mathrm{HC}) \leq 3$ rd percentile for gestational age and sex or $\mathrm{HC}>3$ rd percentile for gestational age and sex and $\mathrm{HC}$ : body length $\leq 0.65$; Laboratory (Lab) criteria: non-negative Zika neutralizing antibody test results in an infant sample. Overall development classification: 1) none: z-scores above -1 SD from the mean on all five ASQ-3 domains, 2) severe: below -2 SD on at least 2 domains, and 3) mild-moderate: the remaining combinations, i.e., z-scores below -2 SD on one domain or from -1 SD to -2 SD on at least one domain. Domain-specific development classification: 1) none: $z$-score above -1 SD from the mean, 2) possible delay (z-score from -1 to $-2 \mathrm{SD})$, and 3 ) likely delay $(z$-score below $-2 \mathrm{SD}) .{ }^{25}$

heterogeneity of functional outcomes at approximately 24 months of age. Those who had microcephaly or disproportionate measurements and met the laboratory criteria were more likely to have multiple, severe functional impairments, and if severely affected, were more likely to have stronger laboratory evidence of possible congenital Zika virus infection than children who met only the laboratory or anthropometric criteria. The group meeting both criteria had the 
most recognizable presentation of congenital Zika infection..$^{5-11}$

Other investigators have reported that children with evidence of prenatal exposure to Zika virus and without birth defects typically have a less severe clinical presentation, although some have seizures, abnormal hearing and vision, and other neurodevelopmental abnormalities. ${ }^{14,15,30,31}$ However, most children in these studies did not have all relevant evaluations, so functional deficits and birth defects may have been under-identified. We found that $5 \%$ of children without physical findings but with some potential laboratory evidence of Zika virus infection had adverse neurologic outcomes and developmental delay. This included one child with the same pattern of severe developmental delay and multiple adverse neurologic outcomes described in the multiply affected group meeting both anthropometric and laboratory criteria, and two children whose only outcomes were mild to moderate developmental delay and seizures. The strength of laboratory evidence for these cases varies, making the correlation between these findings and congenital Zika virus infection challenging.

Others have reported evidence of modest cognitive deficits in infants exposed to Zika virus prenatally who did not have microcephaly. ${ }^{14,32}$ Consistent with these reports, we identified children who had mild to moderate developmental delay without adverse neurologic outcomes among those meeting only the laboratory criteria. However, only one child in this group had laboratory results that met our study definition of a confirmed Zika virus infection. The majority had laboratory results that precluded definitive interpretation because of the inability to distinguish infection from passive transfer of maternal antibodies. The fact that 37 of 100 children with some laboratory evidence of Zika virus infection in our study had neither developmental delay nor adverse neurologic outcomes suggests that either some children infected with Zika virus (perhaps those infected late in gestation) may not develop the functional outcomes we studied or the neutralizing antibodies detected in the children were only from the maternal transfer of these antibodies.

We also explored the possibility that the observed developmental delay could be attributable to causes other than congenital Zika virus infection. Developmental delay, as measured by the ASQ-3 instruments, was common overall in our study population (noted in $62 \%$ of the children, including $55 \%$ of those with no laboratory evidence of infection). Developmental delay was more often severe in children with adverse neurologic manifestations identifiable by the HINE. General estimates of the prevalence of developmental delay in the pediatric population range from $5 \%$ to $10 \%,{ }^{33}$ but the prevalence varies according to case definition and population demographics, including parental education and socioeconomic status. ${ }^{34,35}$

We attempted to adjust for the prevalence of developmental delay by standardizing ASQ-3 scores to the distribution of the scores of Brazilian children from low-income families who received the same questionnaire. The higher prevalence of developmental delay in our study population might in part be explained by the fact that the children we studied were chosen for follow-up because they had anthropometric features or laboratory results that suggested possible congenital Zika infection. We also identified anemia and elevated blood lead levels as possible causes of developmental delay other than possible congenital Zika virus infection in our study population, and the presence of nonspecific dysmorphic features in $22 \%$ of the children with developmental delay may indicate a syndromic diagnosis that could not be explored in this investigation; other prenatal infectious or noninfectious exposures could have affected children's developmental outcomes. Given these potential confounders, it is challenging to know if our results regarding less severe outcomes can be specifically attributed to congenital Zika virus infection.

For children whose only manifestations of congenital infection are developmental delay, early recognition is a challenge. Aragao et al. ${ }^{17}$ used computed tomography (CT) imaging to describe a spectrum of brain damage in congenital Zika infection. However, in many settings, including northeastern Brazil during the 2015-2016 outbreak, imaging may not be readily available, or indicated if available. Furthermore, imaging findings may not be specific. In our study, only 14 of the 43 children with both anthropometric and laboratory evidence of infection had CT imaging results available in medical records. When Zika exposure is suspected, screening for developmental delay, in addition to neuroimaging and $\mathrm{HC}$ measurement, may be necessary to identify affected children for early intervention.

Other investigators have documented features that have expanded the characterization of outcomes of congenital Zika infection, including infants who had typical HCs with nonspecific neurologic signs and isolated eye abnormalities. ${ }^{14,15,17,35}$ We did not find, as other researchers did, that eye abnormalities occurred as the primary or only manifestation of congenital Zika infection. ${ }^{35}$ We did, however, identify four children meeting the laboratory criteria who had retinal findings that are considered pathognomonic for congenital Zika virus infection, ${ }^{36}$ although none had test results indicating confirmed Zika virus infection, by the study definition. Without an ophthalmologic examination, the possible contribution of Zika virus to the medical and developmental outcomes in these children could have been missed.

As for other studies of congenital Zika infection, the main limitations of this investigation were the inability to accurately identify infected children or exposure to maternal infection, to rule out other infectious or noninfectious causes of neurologic disorders and developmental delay, and to obtain complete medical histories from available records. Furthermore, serologic testing was performed when the infants were 17 months of age and residing in areas where there was Zika virus circulation. When laboratory findings suggested infection, we assumed, but were unable to confirm that infection occurred prenatally. This investigation was also limited by small numbers in several categories. Further evaluation is warranted to assess whether comparing Zika-dengue maternal-infant antibody ratios is a valid method for refining interpretation of Zika laboratory test results. Despite these limitations, this study incrementally advances the state of knowledge about the possible consequences of congenital Zika infection by addressing functional outcomes, including developmental delay.

\section{CONCLUSION}

Identifying infants with congenital Zika virus infection remains challenging. Laboratory confirmation of congenital Zika virus infection may not be possible in all infected infants, and anthropometric characteristics are heterogeneous. Because of this, children with congenital Zika infection without obvious clinical manifestations could be missed. When Zika exposure is suspected, ongoing monitoring during the early childhood 
period, including screening for developmental delay in addition to neuroimaging and $\mathrm{HC}$ measurement, may be necessary to identify affected children for early intervention. Questions remain about how closely prenatally exposed children should be monitored and what clinical evaluations are appropriate.

Received December 23, 2019. Accepted for publication February 21, 2020.

Published online March 30, 2020.

Note: Supplemental appendix appears at www.ajtmh.org.

Acknowledgments: We thank the families who participated and the collaborators who assisted with planning and implementing the ZODIAC investigation, and the researchers who shared data from the previous case-control study to support this follow-up investigation.

Financial support: This research was made possible through support provided by the Office of Infectious Diseases, Bureau of Global Health, U.S. Agency for International Development (USAID), under the terms of an Interagency Agreement with the Centers for Disease Control and Prevention (CDC) and Cooperative Agreement number NU2G GH001152. While they contributed to this project, two of the authors were supported by appointments to the Research Participation Program administered by the Oak Ridge Institute for Science and Education through an interagency agreement between the U.S. Department of Energy and CDC.

Disclaimer: The findings and conclusions in this report are those of the authors and do not necessarily represent the official position of the Centers for Disease Control and Prevention.

Authors' addresses: Jeanne Bertolli and Georgina Peacock, Division of Human Development and Disability, Centers for Disease Control and Prevention (CDC), Atlanta, GA, E-mails: jub7@cdc.gov and ghn3@ cdc.gov. Jacob Elijah Attell, Eagle Global Scientific, San Antonio, TX, (now with Booz Allen Hamilton, Inc., McLean, VA), E-mail: attell_ jacob@bah.com. Charles Rose, Cynthia A. Moore, and Coleen A. Boyle, National Center on Birth Defects and Developmental Disabilities, CDC, Atlanta, GA, E-mails: cvr7@cdc.gov, cam0@cdc.gov, and cab3@cdc.gov. Flávio Melo, Hospital Regional de Guarabira/Governo do Estado da Paraíba, João Pessoa, Brazil, E-mail: flaviopediatra@ yahoo.com.br. Jennifer Erin Staples, Division of Vector-Borne Diseases, Centers for Disease Control and Prevention, Fort Collins, CO, E-mail: auv1@cdc.gov. Kim Kotzky and Ashley Satterfield-Nash, Oak Ridge Institute for Science and Education, Oak Ridge, TN, E-mails: kkotzky@gmail.com and yev6@cdc.gov. Nevin Krishna, Office of Public Health Preparedness and Response, CDC, Atlanta, GA, E-mail: hbx0@cdc.gov. Isabela Ornelas Pereira, Transmissible Diseases Department (DEVIT), Ministry of Health of Brazil, Brasilia, Brazil, E-mail: bela.op@gmail.com. André Pessoa, Hospital Infantil Albert Sabin, Fortaleza, Brazil, E-mail: andrepessoa10@yahoo.com.br. Donna Camille Smith, Division of Congenital and Developmental Disorders, CDC, Atlanta, GA, E-mail: dcamillesmith@protonmail.com. Ana Carolina Faria e Silva Santelli, Division of Global HIV and TB, Center for Global Health, CDC, Atlanta, GA, E-mail: nuw6@cdc.gov.

This is an open-access article distributed under the terms of the Creative Commons Attribution (CC-BY) License, which permits unrestricted use, distribution, and reproduction in any medium, provided the original author and source are credited.

\section{REFERENCES}

1. Faria NR et al., 2016. Zika virus in the Americas: early epidemiological and genetic findings. Science 352: 345-349.

2. Brito C, 2015. Zika virus: A new chapter in the history of medicine. Acta Med Port 28: 679-680.

3. Cardoso CW, Paploski JAD, Kikuti M, Rodrigues MS, Silva MM, Campos GS, Sardi SI, Kitron U, Reis MG, Ribeiro GS, 2015. Outbreak of actue exanthematous illness associated with Zika, chikungunya, and dengues viruses, Salvador, Brazil [letter]. Emerg Infect Dis 21: 2274-2276.

4. Pan American Health Organization, 2016. Timeline of Emergence of Zika Virus in the Americas. Available at: http://www.paho.org/ hq/index.php?option=com_content\&view=article\&id=11959: timeline-of-emergence-of-zika-virus-in-the-americas\&ltemid= 41711\&lang=en. Accessed July 15, 2019.

5. Kleber de Oliveira W, Cortez-Escalante J, De Oliveira WT, do Carmo GM, Henriques CM, Coelho GE, Araújo de França GV, 2016. Increase in reported prevalence of microcephaly in infants born to women living in areas with confirmed Zika virus transmission during the first trimester of pregnancy-Brazil, 2015. MMWR Morb Mortal Wkly Rep 65: 242-247.

6. Brasil $P$ et al., 2016. Zika virus infection in pregnant women in rio de Janeiro. N Engl J Med 375: 2321-2334.

7. Rasmussen SA, Jamieson DJ, Honein MA, Petersen LR, 2016. Zika virus and birth defects-reviewing the evidence for causality. N Engl J Med 374: 1981-1987.

8. Moore CA et al., 2017. Characterizing the pattern of anomalies in congenital Zika syndrome for pediatric clinicians. JAMA Pediatr 171: 288-295.

9. Schuler-Faccini L et al.; Brazilian Medical Genetics Society-Zika Embryopathy Task Force, 2016. Possible association between Zika virus infection and microcephaly-Brazil, 2015. MMWR Morb Mortal Wkly Rep 65: 59-62.

10. França GV et al., 2016. Congenital Zika virus syndrome in Brazil: a case series of the first 1501 livebirths with complete investigation. Lancet 388: 891-897.

11. van der Linden $V$ et al., 2016. Description of 13 infants born during October 2015-January 2016 with congenital Zika virus infection without microcephaly at birth - Brazil. MMWR Morb Mortal Wkly Rep 65: 1343-1348.

12. Nogueira ML et al., 2018. Adverse birth outcomes associated with Zika virus exposure during pregnancy in São José do Rio Preto, Brazil. Clin Microbiol Infect 24: 646-652.

13. Moura da Silva AA et al., 2016. Early growth and neurologic outcomes of infants with probable congenital Zika virus syndrome. Emerg Infect Dis J 22: 1953-1956.

14. Nielsen-Saines $\mathrm{K}$ et al., 2019. Delayed childhood neurodevelopment and neurosensory alterations in the second year of life in a prospective cohort of ZIKV-exposed children. Nat Med 25: 1213-1217.

15. Einspieler $C$ et al.; The GM Zika Working Group, 2018. Association of infants exposed to prenatal Zika virus infection with their clinical, neurologic, and developmental status evaluated via the general movement assessment tool. JAMA Netw Open 2: e187235.

16. Oliveira Melo AS, Malinger G, Ximenes R, Szejnfeld PO, Alves Sampaio S, Bispo de Filippis AM, 2016. Zika virus intrauterine infection causes fetal brain abnormality and microcephaly: tip of the iceberg? Ultrasound Obstet Gynecol 47: 6-7.

17. Aragao MFVV et al., 2017. Nonmicrocephalic infants with congenital Zika syndrome suspected only after neuroimaging evaluation compared with those with microcephaly at birth and postnatally: how large is the Zika virus "iceberg"? Am J Neuroradiol 38: 1427-1434.

18. Munoz-Jordan JL, 2017. Diagnosis of Zika virus infections: challenges and opportunities. J Infect Dis 216 (Suppl-10): S951-S956.

19. Wheeler AC, Ventura CV, Ridenour T, Toth D, Nobrega LL, Silva de Souza Dantas LC, Rocha C, Bailey DB Jr, Ventura LO, 2018. Skills attained by infants with congenital Zika syndrome: pilot data from Brazil. PLoS One 13: e0201495.

20. Krow-Lucal ER et al.; Paraíba Microcephaly Work Group, 2018. Association and birth prevalence of microcephaly attributable to Zika virus infection among infants in Paraíba, Brazil, in 2015-16: a case-control study. Lancet Child Adolesc Health 2: 205-213.

21. Walker CL et al., 2018. Femur-sparing pattern of abnormal fetal growth in pregnant women from New York city after maternal Zika virus infection. Am J Obstet Gynecol 219: 187.e1-187.e20.

22. Satterfield-Nash A et al., 2017. Health and development at age 19-24 months of 19 children who were born with microcephaly and laboratory evidence of congenital Zika virus infection during the 2015 Zika virus outbreak-Brazil, 2017. Morb Mortal Wkly Rep 66: 1347-1351.

23. International Fetal and Newborn Growth Consortium for the 21st Century, 2017. Standards for Newborns and References for Very Preterm Infants. Available at: https://intergrowth21.tghn.org/. Accessed July 15, 2019.

24. World Health Organization, 2017. Child Growth Standards. Head Circumference for Age. Available at: http://www.who.int/ 
childgrowth/standards/hc for_age/en/. Accessed July 15, 2019.

25. Maitre NL, Chorna O, Romeo DM, Guzzetta A, 2016. Implementation of the Hammersmith infant neurological exam in a high-risk infant follow-up program. Pediatr Neurol 65: 31-38.

26. Douglass LM et al., 2016. A novel parent questionnaire for the detection of seizures in children. Pediatr Neurol 54: 64-69.e1.

27. Squires J, Twombly E, Bricker D, Potter L, 2009. ASQ-3'M User's Guide, 3rd edition. Baltimore, MD: Paul H. Brooks Publishing Company, Inc., 157-169.

28. Harris PA, Taylor R, Thielke R, Payne J, Gonzalez N, Conde JG, 2009. Research electronic data capture (REDCap) - a metadatadriven methodology and workflow process for providing translational research informatics support. J Biomed Inform 42: 377-381.

29. Filgueiras A, Pires P, Maissonette S, Landeira-Fernandez J, 2013. Psychometric properties of the Brazilian-adapted version of the ages and stages questionnaire in public child daycare centers. Early Hum Dev 89: 561-576.

30. Lopes Moreira ME et al., 2018. Neurodevelopment in infants exposed to Zika virus in utero. N Engl J Med 379: 2377-2379.
31. Rice ME et al., 2018. Vital signs: Zika-associated birth defects and neurodevelopmental abnormalities possibly associated with congenital Zika virus infection-U.S. territories and freely associated states, 2018. MMWR Morb Mortal Wkly Rep 67: 858-867.

32. Valdes $\mathrm{V}$ et al., 2019. Cognitive development of infants exposed to the Zika virus in Puerto Rico. JAMA Netw Open 2: e1914061.

33. Simeonson RJ, Sharp MC, 1992. Developmental delays. Hoekelman RA, Friedman SB, Nelson NM, Seidel HM, eds. Primary Pediatric Care, 2nd edition. St. Louis, MO: C. V. Mosby, 867-870.

34. Gottlieb CA, Maenner MJ, Cappa C, Curkin MS, 2009. Child disability screening, nutrition, and early learning in 18 countries with low and middle incomes: data from the third round of UNICEF's multiple indicator cluster survey (2005-06). Lancet 374: 1831-1839.

35. Durkin M, 2002. The epidemiology of developmental delays in low-income countries. Ment Retard Dev Disabil Res Rev 8: 206-211.

36. Tsui l et al., 2018. Eye findings in infant with suspected or confirmed antenatal Zika virus exposure. Pediatrics 142: e20281104. 